\begin{tabular}{|c|l|}
\hline Title & Nonexistence of Higher Dimensional Stable Turing Patterns in the Singular Limit \\
\hline Author(s) & Nishiura, Y asumasa; Suzuki, Hiromasa \\
\hline Citation & $\begin{array}{l}\text { SIAM Journal on Mathematical A nalysis, 29(5), 1087-1105 } \\
\text { https://doi.org/10.1137/S0036141096313239 }\end{array}$ \\
\hline Issue Date & 1998-09 \\
\hline Doc URL & http://hdl.handle.net/2115/39999 \\
\hline Rights & Copyright $\odot$ 1998 Society for Industrial and A pplied Mathematics \\
\hline Type & article \\
\hline File Information & nishiura 62. pdf \\
\hline
\end{tabular}

Instructions for use 


\title{
NONEXISTENCE OF HIGHER DIMENSIONAL STABLE TURING PATTERNS IN THE SINGULAR LIMIT*
}

\author{
YASUMASA NISHIURA ${ }^{\dagger}$ AND HIROMASA SUZUKI ${ }^{\ddagger}$
}

\begin{abstract}
When the thickness of the interface (denoted by $\varepsilon$ ) tends to zero, any stable stationary internal layered solutions to a class of reaction-diffusion systems cannot have a smooth limiting interfacial configuration. This means that if the limiting configuration of the interface has a smooth limit, it must become unstable for small $\varepsilon$, which makes a sharp contrast with the one-dimensional case. This suggests that stable layered patterns must become very fine and complicated in this singular limit. In fact we can formally derive that the rate of shrinking of stable patterns is of order $\varepsilon^{1 / 3}$. Using this scaling, the resulting rescaled reduced equation determines the morphology of magnified patterns. A variational characterization of the critical eigenvalue combined with the matched asymptotic expansion method is a key ingredient for the proof, although the original linearized system is not of self-adjoint type.
\end{abstract}

Key words. reaction-diffusion system, interfacial pattern, singular perturbation, matched asymptotic expansion

AMS subject classifications. 35B25, 35B35, 35K57, 35R35

PII. S0036141096313239

1. Introduction. Dynamics of interfacial patterns attracts much interest in many fields such as population dynamics, combustion, chemical reaction, solidification, and so on. One of the pioneering works in the pattern formation problem can be traced back to Turing [9], who found that spatially inhomogeneous patterns can be formed by diffusion-driven instability if the inhibitor diffuses faster than the activator. A typical model system is of the form

$$
\begin{cases}u_{t}=\varepsilon^{2} \Delta u+f(u, v), & (x, t) \in \Omega \times(0, \infty), \\ v_{t}=D \Delta v+g(u, v), & (x, t) \in \partial \Omega \times(0, \infty), \\ \frac{\partial u}{\partial n}=0=\frac{\partial v}{\partial n}, & \text {, }\end{cases}
$$

where $u$ is the activator, $v$ is the inhibitor, $\Omega$ is a smooth bounded domain in $\mathbf{R}^{N}$ $(N \geq 2)$, and $\varepsilon$ is a small positive parameter. The nonlinearity $f$ has at least two stable branches for a fixed $v$, and the signs of $g$ are different on these branches, typically $(f, g)=(u(1-u)(u-a)-v, u-\gamma v)$, where $0<a<1, \gamma>0$. More precise assumptions for $(f, g)$ are displayed at the end of this section. Although (1.1) exhibits a variety of patterns depending on diffusion and/or reaction rates, we focus on the stationary ones in higher space dimensions; we are especially interested in layered solutions which have internal transition layers from one stable branch of the nullcline $f=0$ to the other one (see (A.4)). The basic issue asks, Does (1.1) have nonconstant stable stationary solutions up to $\varepsilon=0$ ? And, if it does, what are the

* Received by the editors December 9, 1996; accepted for publication (in revised form) September 15, 1997; published electronically April 14, 1998.

http://www.siam.org/journals/sima/29-5/31323.html

${ }^{\dagger}$ Research Institute for Electronic Science, Hokkaido University, Sapporo 060, Japan (nishiura@aurora.elsip.hokudai.ac.jp).

${ }^{\ddagger}$ Hiroshima National College of Maritime Technology, Higashino-cho, Toyota-gun, Hiroshima, 725-02, Japan (suzuki@hiroshima-cmt.ac.jp). 
asymptotic configurations of them as $\varepsilon \downarrow 0$ ? As we shall see, this is closely related to finding the location of free boundary called the interface separating two different states. Numerically as well as experimentally, for a fixed $\varepsilon>0$, a variety of stationary patterns have been observed such as spots, stripes, and labyrinthine patterns for (1.1) (see, for instance, [1] and the references therein). Hence naively one can expect that (1.1) has a lot of stable stationary solutions for small $\varepsilon$ up to $\varepsilon=0$.

In fact, for the one-dimensional case, it is proved rigorously (see [5]) that many stable layered solutions coexist up to $\varepsilon=0$. It should be noted that each layer position has a definite limit and the distance between layer positions remains finite as $\varepsilon \downarrow 0$.

On the other hand, for the higher dimensional case, we know very little about the limiting configuration of stable stationary solutions to (1.1) when $\varepsilon$ tends to zero. For instance, the planar front does not persist as a stable one (see [7]), and more complicated patterns take it over for small $\varepsilon$. We rephrase our basic question in the following way: Does (1.1) have an $\varepsilon$-family of stable stationary layered solutions $\left(U^{\varepsilon}, V^{\varepsilon}\right)$ with smooth interface $\Gamma^{\varepsilon}$ up to $\varepsilon=0$ ? Here $\Gamma^{\varepsilon}$ is defined by

$$
\Gamma^{\varepsilon} \equiv\left\{x \in \Omega \mid U^{\varepsilon}(x)=\alpha^{*}\right\}
$$

where $\alpha^{*}$ is an intermediate value between two stable branches of $f=0$ and, for instance, is equal to $1 / 2$ for the above specific example. Note that "smooth up to $\varepsilon=0$ " means that there exists an $(N-1)$-dimensional smooth compact connected manifold $\Gamma^{0}$ without boundary embedded in $\mathbf{R}^{N}$ such that $\Gamma^{\varepsilon}$ converges to $\Gamma^{0}$ smoothly as $\varepsilon \downarrow 0$.

The goal in this paper is to give a negative answer to this question under the assumption that it has a matched asymptotic expansion of order 1 (see section 2 for details). Namely, we have the following theorem.

THEOREM 1.1 (main theorem). Suppose that (1.1) has an $\varepsilon$-family of stationary matched asymptotic solutions of order 1 whose interface is smooth up to $\varepsilon=0$. Then it must become unstable for small $\varepsilon$.

We prove this in section 3 by converting the linearized eigenvalue problem around $\left(U^{\varepsilon}, V^{\varepsilon}\right)$ to a variational one (see (3.5)) which consists of a scalar elliptic part and a nonlocal term coming from the coupling of the two equations. A key idea is that in the variational characterization of the maximal eigenvalue of the system, the term associated with the (local) elliptic boundary problem is positive and it dominates the contribution from the nonlocal term for a highly oscillating admissible function in azimuthal direction. This instability result leads to the natural question, how about the fate of stable ones when $\varepsilon \downarrow 0$ ? The above theorem strongly suggests that stable patterns somehow must become very fine and/or complicated when $\varepsilon \downarrow 0$, and if it happens, can we characterize the domain size of those patterns and their morphologies? We shall discuss these issues in section 4 ; in fact we can formally derive that the rate of shrinking of stable patterns is of order $\varepsilon^{1 / 3}$. Using this scaling, the resulting rescaled reduced equation determines the morphology of magnified patterns (see also [5], [6], and [8]).

We prove the above theorem under the following assumptions.

(A.0) $\Gamma^{\varepsilon}$ is an $(N-1)$-dimensional smooth compact connected manifold without boundary inside of $\Omega$, and the domain surrounded by $\Gamma^{\varepsilon}$ is simply connected.

(A.1) $f$ and $g$ are smooth functions of $u$ and $v$ defined on some open set $\mathcal{O}$ in $\mathbf{R}^{2}$ and the partial derivative $f_{v}$ (resp., $g_{u}$ ) is a negative (resp., positive) constant function.

(A.2) (a) The nullcline of $f$ is sigmoidal and consists of three smooth curves $u=h_{-}(v), h_{0}(v)$, and $h_{+}(v)$ defined on the intervals $I_{-}, I_{0}$, and $I_{+}$, respectively. Let 


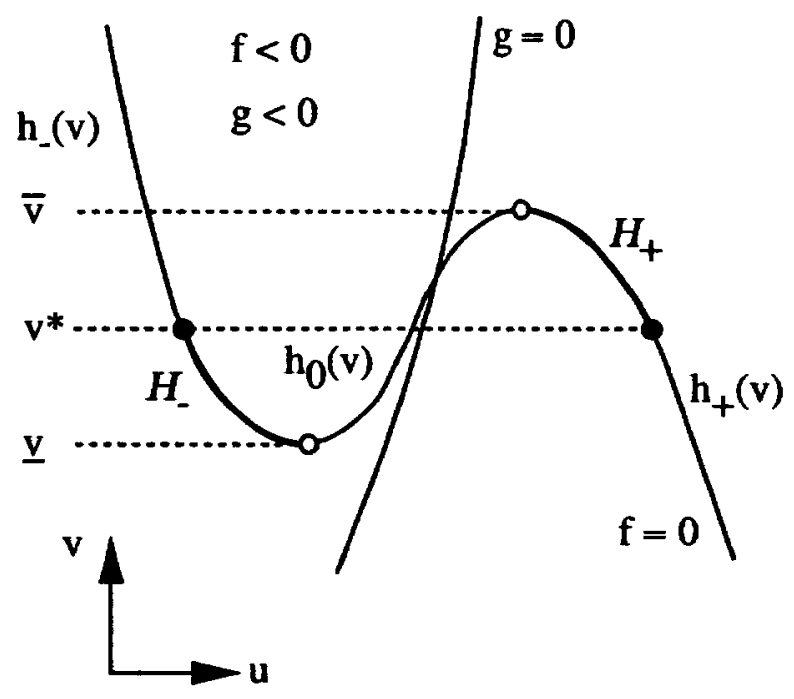

FIG. 1.1. Functional forms of $f=0$ and $g=0$.

$\min I_{-}=\underline{v}$ and $\max I_{+}=\bar{v}$; then the inequality $h_{-}(v)<h_{0}(v)<h_{+}(v)$ holds for $v \in I^{*} \equiv(\underline{v}, \bar{v})$, and $h_{+}(v)$ (resp., $\left.h_{-}(v)\right)$ coincides with $h_{0}(v)$ at only one point $v=\bar{v}$ (resp., $\underline{v})$, respectively.

(b) The nullcline of $g$ intersects with that of $f$ at one or three points transversally as in Figure 1.1. The critical point on $u=h_{-}(v)$ (resp., $h_{+}(v)$ or $\left.h_{0}(v)\right)$, if it exists, is denoted by $P=\left(u_{-}, v_{-}\right)=\left(h_{-}\left(v_{-}\right), v_{-}\right)$(resp., $Q=\left(u_{+}, v_{+}\right)=\left(h_{+}\left(v_{+}\right), v_{+}\right)$or $\left.R=\left(u_{0}, v_{0}\right)=\left(h_{0}\left(v_{0}\right), v_{0}\right)\right)$.

(A.3) $J(v)$ has an isolated zero at $v=v^{*} \in I^{*}$ such that $d J / d v<0$ at $v=v^{*}$, where $J(v)=\int_{h_{-}(v)}^{h_{+}(v)} f(s, v) d s$. Moreover, we assume that $v_{-}<v^{*}<v_{+}$.

(A.4) $f_{u}<0$ on $H_{+} \cup H_{-}$, where $H_{-}$(resp., $H_{+}$) denotes the part of the curve $u=$ $h_{-}(v)\left(\right.$ resp.,$\left.h_{+}(v)\right)$ defined by $H_{-}\left(\right.$resp.,$\left.H_{+}\right)=\left\{(u, v) \mid u=h_{-}(v)\left(\right.\right.$ resp.,$\left.h_{+}(v)\right)$ for $\left.v_{-} \leq v<v^{*}\left(v^{*}<v \leq v_{+}\right)\right\}$, respectively. Note that $v_{-} \leq$(resp., $\left.\leq v_{+}\right)$is replaced by $\underline{v}<$ (resp., $<\bar{v}$ ) when there are no critical points on the branch $u=h_{-}(v)$ (resp., $\left.h_{+}(v)\right) . H_{+}$and $H_{-}$are called the stable branches of $f=0$. See the thick solid part of $f=0$ in Figure 1.1.

$$
\begin{gathered}
\left.g\right|_{H_{-}}<0<\left.g\right|_{H_{+}}, \\
\left.\operatorname{det}\left(\frac{\partial(f, g)}{\partial(u, v)}\right)\right|_{H_{+} \cup H_{-}}>0 . \\
\left.g_{v}\right|_{H_{+} \cup H_{-}}<0 .
\end{gathered}
$$

Remark 1.1. The assumption for the partial derivatives in (A.1) is necessary to make the problem (3.4) self-adjoint; however, it is plausible that our result holds true for the general case.

Remark 1.2. It is not known rigorously that, under the above assumptions, any smooth layered solution with a smooth limiting interface has a matched asymptotic 
expansion of order 1; however, it is conjectured to be true at least for a large class of such layered solutions.

Remark 1.3. (A.4) and (A.5) (b) imply that

$$
\frac{d}{d v} g\left(h_{ \pm}(v), v\right)=\left.\frac{f_{u} g_{v}-f_{v} g_{u}}{f_{u}}\right|_{H_{+} \cup H_{-}}<0 .
$$

We use the following notation throughout the paper: let $\sigma=\left(\sigma_{1}, \sigma_{2}, \ldots, \sigma_{N}\right)$ denote the usual multi-index of order $|\sigma|=\sigma_{1}+\sigma_{2}+\cdots+\sigma_{N}$ with nonnegative integers $\sigma_{i}$, and write $\partial_{i}=\partial / \partial x_{i}(1 \leq i \leq N)$.

(i) Let $k$ be a nonnegative integer and $\alpha \in(0,1)$. By $C^{k+\alpha}(\bar{\Omega})$ we mean the Banach space of all real-valued functions $u \in C^{k}(\bar{\Omega})$ for which the derivatives $\partial^{\sigma} u$ with $|\sigma|=k$ are Hölder continuous on $\bar{\Omega}$ with exponent $\alpha$. The norm is

$$
\|u\|_{C^{k+\alpha}(\bar{\Omega})}=\sum_{j=0}^{k}|u|_{j, \bar{\Omega}}+|u|_{k+\alpha, \bar{\Omega}},
$$

where

$$
|u|_{j, \bar{\Omega}}=\max _{|\sigma|=k} \sup _{x \in \bar{\Omega}}\left|\partial^{\sigma} u(x)\right|
$$

and

$$
|u|_{k+\alpha, \bar{\Omega}}=\max _{|\sigma|=k} \sup _{x, y \in \bar{\Omega}} \frac{\left|\partial^{\sigma} u(x)-\partial^{\sigma} u(y)\right|}{|x-y|^{\alpha}} .
$$

(ii) $C_{0}^{k+\alpha}(\bar{\Omega})$ is the subspace of $C^{k+\alpha}(\bar{\Omega})$ whose elements are functions vanishing on $\partial \Omega$.

(iii) $C_{\varepsilon}^{k+\alpha}(\bar{\Omega})$ is the Banach space of all real-valued functions in $C^{k+\alpha}(\bar{\Omega})$ but with the special norm depending on $\varepsilon$ :

$$
\|u\|_{C_{\varepsilon}^{k+\alpha}(\bar{\Omega})}=\sum_{j=0}^{k} \varepsilon^{j}|u|_{j, \bar{\Omega}}+\varepsilon^{k+\alpha}|u|_{k+\alpha, \bar{\Omega}} .
$$

(iv) $C_{\varepsilon, 0}^{k+\alpha}(\bar{\Omega})$ is the subspace of $C_{\varepsilon}^{k+\alpha}(\bar{\Omega})$ whose elements are functions vanishing on $\partial \Omega$.

2. Matched asymptotic expansion of singularly perturbed stationary solutions. In this section, we summarize the necessary conditions for the existence of the $\varepsilon$-family of matched asymptotic solutions with internal transition layers of the following stationary problem:

$$
\begin{array}{ll} 
\begin{cases}0=\varepsilon^{2} \Delta u+f(u, v) & \text { in } \Omega, \\
0=D \Delta v+g(u, v) & \text { on } \partial \Omega .\end{cases} \\
\frac{\partial u}{\partial n}=0=\frac{\partial v}{\partial n}
\end{array}
$$

Before presenting the precise form of matched asymptotic expansion, we need to do a change of variables near the interface. Let us assume that there exists an $\varepsilon$-family 
of smooth solutions $\left(U^{\varepsilon}(x), V^{\varepsilon}(x)\right)$ to $(2.1)$ and (2.2) with interior transition layers such that the interface defined by

$$
\Gamma^{\varepsilon} \equiv\left\{x \in \Omega \mid U^{\varepsilon}(x)=\alpha^{*}\right\}
$$

is a compact smooth manifold of dimension $N-1$ embedded in $\mathbf{R}^{N}$ and has a definite limit $\Gamma^{0}$ with the same properties as $\varepsilon \downarrow 0$. Let $\left(X_{\phi}, \phi\right)$ be a local chart on $\Gamma^{0}$, with $\phi\left(X_{\phi}\right)$ an open subset of $\mathbf{R}^{N-1}$. For $x_{0} \in X_{\phi}, \phi\left(x_{0}\right)=s=\left(s^{1}, \ldots, s^{N-1}\right)$ and we denote the inverse of $\phi$ by

$$
x_{0}=\left(x_{0}^{1}(s), \ldots, x_{0}^{N}(s)\right) .
$$

In some tubular neighborhood $U_{d}\left(\Gamma^{0}\right)=\left\{x \in \mathbf{R}^{N}|| y(x) \mid \leq d\right\}$ of $\Gamma^{0}$, the local coordinate system $(s, y)=\left(s^{1}, \ldots, s^{N-1}, y\right)$ is defined and for $x \in U_{d}\left(\Gamma^{0}\right)$,

$$
x=X(s, y) \equiv x_{0}\left(s^{1}, \ldots, s^{N-1}\right)+y \nu\left(s^{1}, \ldots, s^{N-1}\right)
$$

holds, where $\nu\left(s^{1}, \ldots, s^{N-1}\right)$ is the outward unit normal vector at $s=\left(s^{1}, \ldots, s^{N-1}\right)$ to $\Gamma^{0}$. Then, $X$ becomes a diffeomorphism from $[-d, d] \times \Gamma^{0}$ to $U_{d}\left(\Gamma^{0}\right)$ if $d$ is strictly smaller than the infimum of the radii of curvature of $\Gamma^{0}$. Its inverse is denoted by $(S(x), Y(x))$. Then $\Gamma^{\varepsilon}$ can be represented by

$$
\Gamma^{\varepsilon}=\left\{x_{0}(s)+\gamma(s, \varepsilon) \nu(s) \mid s \in \Gamma^{0}\right\},
$$

where

$$
\gamma(s, \varepsilon)=\sum_{k=1}^{m} \varepsilon^{k} \gamma_{k}(s)+\varepsilon^{m} \hat{\gamma}_{m+1}(s, \varepsilon) .
$$

Here we introduce the local shift variable $\tau$ by the following relation:

$$
y=\tau+\omega\left(\frac{\tau}{d}\right) \gamma(s, \varepsilon),
$$

where $\omega(\tau) \in C^{\infty}(\mathbf{R})$ is a cut-off function such that

$$
\begin{gathered}
\omega(\tau)=1 \text { for }|\tau| \leq \frac{1}{2}, \omega(\tau)=0 \text { for }|\tau| \geq 1, \\
0 \leq \omega(\tau) \leq 1, \quad\left|\omega^{\prime}\right| \leq 3 .
\end{gathered}
$$

Then, by the implicit function theorem, $\tau=\tau(s, y, \varepsilon)$ satisfying (2.5) is defined for sufficiently small $\varepsilon$. In place of $x$, we use a new independent variable $\hat{x}$, defined by

$$
\hat{x}=\hat{X}(x, \varepsilon)= \begin{cases}x, & x \in \Omega \backslash U_{d}\left(\Gamma^{0}\right), \\ X(S(x), \tau(S(x), Y(x), \varepsilon)), & x \in U_{d}\left(\Gamma^{0}\right) .\end{cases}
$$

Let $\Omega_{\varepsilon}^{+}$(resp., $\Omega_{0}^{+}$) be the region surrounded by $\Gamma^{\varepsilon}$ (resp., $\Gamma^{0}$ ) and $\Omega_{\varepsilon}^{-} \equiv \Omega \backslash \bar{\Omega}_{\varepsilon}^{+}$ (resp., $\left.\Omega_{0}^{-} \equiv \Omega \backslash \bar{\Omega}_{0}^{+}\right)$. Then, note that $\hat{x}=\hat{X}(x, \varepsilon)$ maps $\Gamma^{\varepsilon}$ to $\Gamma^{0}$, and $\Omega_{\varepsilon}^{ \pm}$to $\Omega_{0}^{ \pm}$, respectively; namely, the free boundary $\Gamma^{\varepsilon}$ becomes a fixed boundary $\Gamma^{0}$ in the new coordinate. Throughout the paper, we shall use the following notation:

$$
u(x)=u(s, y), \quad \hat{u}(\hat{x})=\hat{u}(s, \tau) .
$$


Using the above transformation, the stationary problem (2.1) with (2.2) can be rewritten as

$$
\begin{cases}\varepsilon^{2} M^{\varepsilon} \hat{u}+f(\hat{u}, \hat{v})=0 & \text { in } \Omega, \\ D M^{\varepsilon} \hat{v}+g(\hat{u}, \hat{v})=0 & \\ \frac{\partial \hat{u}}{\partial n}=0=\frac{\partial \hat{v}}{\partial n} & \text { on } \partial \Omega,\end{cases}
$$

where $\hat{u}=\hat{u}(\hat{x}), \hat{v}=\hat{v}(\hat{x})$, and $M^{\varepsilon}$ is the representation of the Laplacian $\Delta_{x}$ in $\hat{x}$. In $\Omega \backslash U_{d}\left(\Gamma^{0}\right), M^{\varepsilon}$ is equal to $\Delta_{\hat{x}}$. On the other hand, in the neighborhood $U_{d}\left(\Gamma^{0}\right)$, $M^{\varepsilon}$ is defined in the following way: for the local coordinate system $(s, y)$ defined by (2.4) in $\mathbf{R}^{N}$, let $g^{i j}$ be the contravariant metric tensor and $g=\operatorname{det}\left(g^{i j}\right)$. Then for $u(x)=u(s, y)$, Laplacian $\Delta_{x}$ is expressed by

$$
\begin{aligned}
\left(\Delta_{x} u\right)(x)= & \left(\Delta_{(s, y)} u\right)(s, y) \\
\equiv & \frac{\partial^{2}}{\partial y^{2}} u(s, y)+(N-1) H(s, y) \frac{\partial}{\partial y} u(s, y) \\
& \quad+\frac{1}{\sqrt{g}} \sum_{i=1}^{N-1} \frac{\partial}{\partial s^{i}}\left(\sqrt{g} \sum_{j=1}^{N-1} g^{i j} \frac{\partial}{\partial s^{j}} u(s, y)\right),
\end{aligned}
$$

where $H=H(s, y)$ is the mean curvature of the hypersurface $\Gamma(y)=\left\{x_{0}(s)+y \nu(s) \mid\right.$ $\left.s \in \Gamma^{0}\right\}$ at $(s, y)$. Using this representation, for $\hat{u}(\hat{x})=\hat{u}(s, \tau), M^{\varepsilon}$ is defined by

$$
\left(M^{\varepsilon} \hat{u}\right)(\hat{x}) \equiv \Delta_{(s, y)} \hat{u}(s, \tau(s, y, \varepsilon)) .
$$

It follows from this definition that $M^{\varepsilon}$ can be expanded as $M^{\varepsilon}=\sum_{k \geq 0} \varepsilon^{k} M_{k}$, where

$$
M_{0} \equiv \Delta_{\hat{x}}, \quad \hat{x} \in \Omega,
$$

and for $k \geq 1$,

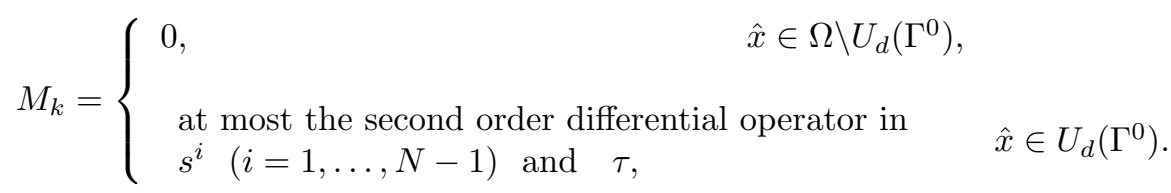

In the following, we consider only (2.6) and (2.7), so we omit the hat symbol $\left(^{\wedge}\right)$. A family of solutions $\left(U^{\varepsilon}, V^{\varepsilon}\right) \in X_{\varepsilon} \equiv C_{\varepsilon}^{2+\alpha}(\bar{\Omega}) \times C^{2+\alpha}(\bar{\Omega})$ of (2.6) and (2.7) is called an $\varepsilon$-family of matched asymptotic solutions of order $m$ when it has the following expansions (2.9)-(2.12). Roughly speaking, $\left(U^{\varepsilon}, V^{\varepsilon}\right)$ is expanded separately in two regions $\Omega_{0}^{ \pm}$divided by the interface $\Gamma^{0}$, and they are matched smoothly at $\Gamma^{0}$. It should be recalled that the boundary condition $(2.3)$ is always satisfied at $\Gamma^{0}$ for small $\varepsilon$ owing to the change of variables (2.5). More precisely, for any positive integer $m$, we have

$$
U^{\varepsilon}(x)= \begin{cases}U_{+}^{\varepsilon}(x) \equiv U_{m}^{+}(x, \varepsilon)+\Phi_{m}^{+}(x, \varepsilon)+o\left(\varepsilon^{m}\right), & x \in \Omega_{0}^{+}, \\ U_{-}^{\varepsilon}(x) \equiv U_{m}^{-}(x, \varepsilon)+\Phi_{m}^{-}(x, \varepsilon)+o\left(\varepsilon^{m}\right), & x \in \Omega_{0}^{-},\end{cases}
$$




$$
V^{\varepsilon}(x)= \begin{cases}V_{+}^{\varepsilon}(x) \equiv V_{m}^{+}(x, \varepsilon)+\varepsilon^{2} \Psi_{m}^{+}(x, \varepsilon)+o\left(\varepsilon^{m}\right), & x \in \Omega_{0}^{+}, \\ V_{-}^{\varepsilon}(x) \equiv V_{m}^{-}(x, \varepsilon)+\varepsilon^{2} \Psi_{m}^{-}(x, \varepsilon)+o\left(\varepsilon^{m}\right), & x \in \Omega_{0}^{-},\end{cases}
$$

where

$$
\begin{gathered}
U_{m}^{ \pm}(x, \varepsilon)=\sum_{k=0}^{m} u_{k}^{ \pm}(x) \varepsilon^{k}, \quad V_{m}^{ \pm}(x, \varepsilon)=\sum_{k=0}^{m} v_{k}^{ \pm}(x) \varepsilon^{k}, \\
\Phi_{m}^{ \pm}(x, \varepsilon)= \begin{cases}\omega\left(\frac{Y(x)}{d}\right) \sum_{k=0}^{m} \phi_{k}^{ \pm}\left(S(x), \frac{Y(x)}{\varepsilon}\right) \varepsilon^{k}, & x \in U_{d}\left(\Gamma^{0}\right) \cap \Omega_{0}^{ \pm}, \\
0, & x \in \Omega_{0}^{ \pm} \backslash U_{d}\left(\Gamma^{0}\right),\end{cases} \\
\Psi_{m}^{ \pm}(x, \varepsilon)= \begin{cases}\omega\left(\frac{Y(x)}{d}\right) \sum_{k=0}^{m} \psi_{k}^{ \pm}\left(S(x), \frac{Y(x)}{\varepsilon}\right) \varepsilon^{k}, & x \in U_{d}\left(\Gamma^{0}\right) \cap \Omega_{0}^{ \pm}, \\
0, & x \in \Omega_{0}^{ \pm} \backslash U_{d}\left(\Gamma^{0}\right),\end{cases}
\end{gathered}
$$

$\phi_{k}^{ \pm}$and $\psi_{k}^{ \pm}$are functions of $s$ and $\xi$, and $\xi$ is the stretched variable $\xi \equiv \tau / \varepsilon$ (recall that $Y(\hat{x})=\tau$ ). Here the topology of Landau's $o$ is $X_{\varepsilon}$. The coefficients $u_{k}^{ \pm}, v_{k}^{ \pm}, \phi_{k}^{ \pm}$, and $\psi_{k}^{ \pm}$satisfy the equations listed below in appropriate function spaces, which can be obtained by making outer and inner expansions and equating like powers of $\varepsilon^{k}$. The inner and outer solutions are not independent in the sense that they must satisfy the boundary conditions as well as the $C^{1}$-matching conditions between $\left(U_{+}^{\varepsilon}, V_{+}^{\varepsilon}\right)$ and $\left(U_{-}^{\varepsilon}, V_{-}^{\varepsilon}\right)$ on $\Gamma^{0}$. Let $\beta^{\varepsilon}(s)=v^{*}+\sum_{k=1}^{m} \beta_{k}(s) \varepsilon^{k}+\varepsilon^{m} \beta_{m+1}(s, \varepsilon)$ be the expansion of the value of $V^{\varepsilon}$ on $\Gamma^{0}$. Note that the 0 th order should be $v^{*}$ from (A.3), since $\left(U^{\varepsilon}, V^{\varepsilon}\right)$ is a stationary solution.

We briefly explain the algorithm of the matched asymptotic expansion method and display the equations and relations up to order $O\left(\varepsilon^{m}\right)$. For more detailed arguments, see [2] and [6].

First we divide (2.6) into two problems as follows:

$$
\begin{aligned}
& \begin{cases}\varepsilon^{2} M^{\varepsilon} u^{+}+f\left(u^{+}, v^{+}\right)=0 & \text { in } \Omega_{0}^{+}, \\
D M^{\varepsilon} v^{+}+g\left(u^{+}, v^{+}\right)=0 & \\
u^{+}=\alpha^{*}, v^{+}=\beta^{\varepsilon} & \text { on } \Gamma^{0},\end{cases} \\
& \begin{cases}\varepsilon^{2} M^{\varepsilon} u^{-}+f\left(u^{-}, v^{-}\right)=0 & \text { in } \Omega_{0}^{-}, \\
D M^{\varepsilon} v^{-}+g\left(u^{-}, v^{-}\right)=0 & \text { on } \Gamma^{0}, \\
u^{-}=\alpha^{*}, v^{-}=\beta^{\varepsilon} & \text { on } \partial \Omega . \\
\frac{\partial u^{-}}{\partial n}=0=\frac{\partial v^{-}}{\partial n} & \text {. }\end{cases}
\end{aligned}
$$

Then the interface is regarded as the boundary layer at $\Gamma^{0}$. 
Outer expansion. Let

$$
u^{ \pm}=\sum_{k=0}^{m} u_{k}^{ \pm}(x) \varepsilon^{k}, \quad v^{ \pm}=\sum_{k=0}^{m} v_{k}^{ \pm}(x) \varepsilon^{k}
$$

where both $u_{k}^{ \pm}(x)$ and $v_{k}^{ \pm}(x)$ belong to $C^{\infty}\left(\bar{\Omega}_{0}^{ \pm}\right)$. Substituting (2.14) into (2.13) \pm and equating like powers of $\varepsilon$, then $\left(u_{k}^{ \pm}(x), v_{k}^{ \pm}(x)\right)$ satisfy the following equations:

$k=0$

$$
\begin{cases}f\left(u_{0}^{ \pm}, v_{0}^{ \pm}\right)=0 & \text { in } \Omega_{0}^{ \pm} \\ D M_{0} v_{0}^{ \pm}+g\left(u_{0}^{ \pm}, v_{0}^{ \pm}\right)=0 & \text { on } \partial \Omega \\ \frac{\partial v_{0}^{-}}{\partial n}=0 & \end{cases}
$$

$k \geq 1$

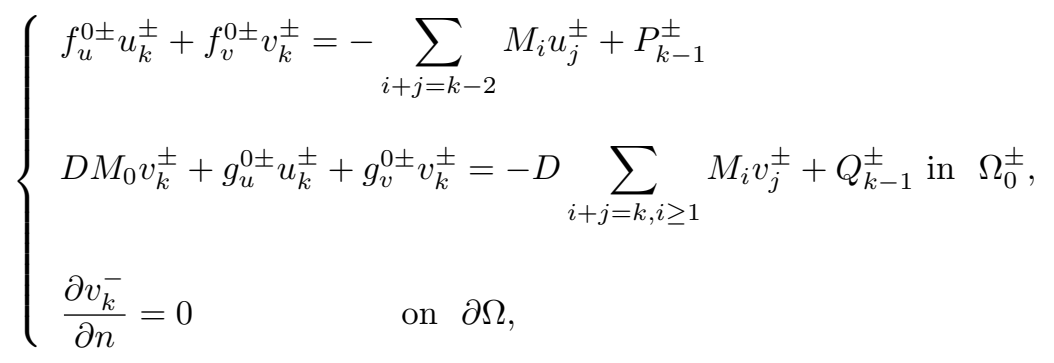

where $f_{u}^{0 \pm} \equiv \frac{\partial}{\partial u} f\left(u_{0}^{ \pm}, v_{0}^{ \pm}\right), f_{v}^{0 \pm} \equiv \frac{\partial}{\partial v} f\left(u_{0}^{ \pm}, v_{0}^{ \pm}\right)$, and so on. $P_{k-1}^{ \pm}$and $Q_{k-1}^{ \pm}$are functions determined only by $u_{0}^{ \pm}, v_{0}^{ \pm}, \ldots, u_{k-1}^{ \pm}, v_{k-1}^{ \pm}$. For $k=1$, we define the righthand side of (2.16) as equal to zero. Since $\left(U^{\varepsilon}, V^{\varepsilon}\right)$ is a layered solution connecting two stable branches of the kinetics $(f, g)$, it follows from (A.4) and the first equation of (2.15) that $\left(u_{0}^{ \pm}, v_{0}^{ \pm}\right)$lies on either $H_{+}$or $H_{-}$. For definiteness, we assume that $u_{0}^{ \pm}=$ $h_{ \pm}\left(v_{0}^{ \pm}\right)$. Then $(2.16)$ can be uniquely solved recursively owing to the assumptions (A.4) and (A.5). This expansion is, however, insufficient because the layer part is not taken into account; in fact, $u_{0}^{+}$and $u_{0}^{-}$are discontinuous on $\Gamma^{0}$. To cope with this defect, we introduce a new variable $\xi=\tau / \varepsilon$ that rescales a neighborhood of the interface. Also note that the boundary conditions for $v_{k}^{ \pm}$are determined by the $C^{1}$ matching conditions discussed later.

Inner expansion. We introduce the stretched variable $\xi=\tau / \varepsilon$ and let

$$
\begin{aligned}
& u^{ \pm}=U_{m}^{ \pm}(x, \varepsilon)+\sum_{k=0}^{m} \phi_{k}^{ \pm}\left(S(x), \frac{Y(x)}{\varepsilon}\right) \varepsilon^{k}, \\
& v^{ \pm}=V_{m}^{ \pm}(x, \varepsilon)+\varepsilon^{2} \sum_{k=0}^{m} \psi_{k}^{ \pm}\left(S(x), \frac{Y(x)}{\varepsilon}\right) \varepsilon^{k},
\end{aligned}
$$

where $\phi_{k}^{ \pm}=\phi_{k}^{ \pm}(s, \xi)$ and $\psi_{k}^{ \pm}=\psi_{k}^{ \pm}(s, \xi)$. Since the definition domains of $\phi_{k}^{ \pm}$and $\psi_{k}^{ \pm}$are semi-infinite, these functions and the inhomogeneous terms of their equations listed below must have some decaying property for solvability. An appropriate function space for this purpose is the following. 
Definition 2.1. Let $\mathcal{E}^{ \pm}$be the set of functions $E^{ \pm}(s, \xi, \varepsilon)$ defined on $\Gamma_{0} \times I^{\mp} \times$ $\left[0, \varepsilon_{0}\right)$ with the property that for each $C^{\infty}$ linear differential operator $D$ of any order in the variables $s$ and $\xi$, there exist positive constants $C_{ \pm}$and $K$ (possibly depending on $D$ and $E^{ \pm}$, but not on $s, \xi$, and $\left.\varepsilon\right)$ with $\left|D E^{ \pm}\right| \leq K e^{-C_{ \pm}|\xi|}$. Here $I^{-} \equiv(-\infty, 0)$ and $I^{+} \equiv(0, \infty)$.

Substituting (2.17) into $(2.13)_{ \pm}$and equating like powers of $\varepsilon$, we obtain the following equations:

$k=0$

$$
\left\{\begin{array}{l}
\ddot{\phi}_{0}^{ \pm}+f\left(h_{ \pm}\left(v^{*}\right)+\phi_{0}^{ \pm}, v^{*}\right)=0, \\
D \ddot{\psi}_{0}^{ \pm}=g\left(h_{ \pm}\left(v^{*}\right), v^{*}\right)-g\left(h_{ \pm}\left(v^{*}\right)+\phi_{0}^{ \pm}, v^{*}\right), \\
\phi_{0}^{ \pm}(s, \mp \infty)=0, \quad \psi_{0}^{ \pm}(s, \mp \infty)=0=\dot{\psi}_{0}^{ \pm}(s, \mp \infty),
\end{array}\right.
$$

$k=1$

$$
\left\{\begin{array}{c}
\ddot{\phi}_{1}^{ \pm}+\tilde{f}_{u}^{0 \pm} \phi_{1}^{ \pm}=-\tilde{M}_{1} \phi_{0}^{ \pm}-\tilde{f}_{u}^{0 \pm}\left\{u_{1}^{ \pm}(s, 0)+\left(u_{0}^{ \pm}\right)_{\tau}(s, 0) \xi\right\} \\
-\tilde{f}_{v}^{0 \pm}\left\{v_{1}^{ \pm}(s, 0)+\left(v_{0}^{ \pm}\right)_{\tau}(s, 0) \xi\right\} \\
D \ddot{\psi}_{1}^{ \pm}=-D \tilde{M}_{1} \psi_{0}^{ \pm}+\tilde{Q}_{0}^{ \pm}, \\
\phi_{1}^{ \pm}(s, \mp \infty)=0, \quad \psi_{1}^{ \pm}(s, \mp \infty)=0=\dot{\psi}_{1}^{ \pm}(s, \mp \infty),
\end{array}\right.
$$

$k \geq 2$

$$
\left\{\begin{array}{l}
\ddot{\phi}_{k}^{ \pm}+\tilde{f}_{u}^{0 \pm} \phi_{k}^{ \pm}=-\sum_{i+j=k, i \geq 1} \tilde{M}_{i} \phi_{j}^{ \pm}+\tilde{P}_{k-1}^{ \pm}, \\
D \ddot{\psi}_{k}^{ \pm}=-D \sum_{i+j=k, i \geq 1} \tilde{M}_{i} \psi_{j}^{ \pm}+\tilde{Q}_{k-1}^{ \pm}, \\
\phi_{k}^{ \pm}(s, \mp \infty)=0, \quad \psi_{k}^{ \pm}(s, \mp \infty)=0=\dot{\psi}_{k}^{ \pm}(s, \mp \infty)
\end{array}\right.
$$

for $\xi \in I^{\mp}$ and $s \in \Gamma^{0}$, where $\cdot=\frac{\partial}{\partial \xi}, \tilde{f}_{u}^{0 \pm} \equiv \frac{\partial}{\partial u} f\left(h_{ \pm}\left(v^{*}\right)+\phi_{0}^{ \pm}, v^{*}\right),\left(u_{0}^{ \pm}\right)_{\tau}(s, 0)=$ $\frac{\partial}{\partial \tau} u_{0}^{ \pm}(s, 0)$, and so on. $\tilde{P}_{k-1}^{ \pm}$depends on $u_{0}^{ \pm}, v_{0}^{ \pm}, \ldots, u_{k}^{ \pm}, v_{k}^{ \pm}, \phi_{0}^{ \pm}, \psi_{0}^{ \pm}, \ldots, \phi_{k-1}^{ \pm}, \psi_{k-2}^{ \pm}$, and $\tilde{Q}_{k-1}^{ \pm}$does, moreover, depend on $\psi_{k-1}^{ \pm}$. The solvability of the above equations in the space $\mathcal{E}^{ \pm}$can be shown in a similar way to [2], so we leave the details to the reader. $\tilde{M}^{\varepsilon}$ is the representation of $M^{\varepsilon}$ in variables $s$ and $\xi$ and is expanded as

$$
\tilde{M}^{\varepsilon} \equiv \frac{1}{\varepsilon^{2}} \sum_{k \geq 0} \varepsilon^{k} \tilde{M}_{k}
$$

Here $\tilde{M}_{k}(k \geq 0)$ are at most second-order differential operators in $s$ and $\xi$. The precise forms of $\tilde{M}_{k}$ are presented at the end of this section.

Boundary conditions and $C^{1}$-matching conditions. Now we describe the boundary conditions of $v_{k}^{ \pm}$and $\phi_{k}^{ \pm}$on $\Gamma^{0}$. Then $u_{k}^{ \pm}, v_{k}^{ \pm}, \phi_{k}^{ \pm}$, and $\psi_{k}^{ \pm}$are determined 
recursively. These conditions are given by

$$
\begin{aligned}
& \alpha^{*}=\sum_{k=0}^{m} u_{k}^{ \pm}(s, 0) \varepsilon^{k}+\sum_{k=0}^{m} \phi_{k}^{ \pm}(s, 0) \varepsilon^{k} \\
& v^{*}+\sum_{k=1}^{m} \beta_{k}(s) \varepsilon^{k}=\sum_{k=0}^{m} v_{k}^{ \pm}(s, 0) \varepsilon^{k}+\varepsilon^{2} \sum_{k=0}^{m-2} \psi_{k}^{ \pm}(s, 0) \varepsilon^{k} .
\end{aligned}
$$

Equating like powers of $\varepsilon$, we have the following boundary conditions:

$$
k=0
$$

$$
\phi_{0}^{ \pm}(s, 0)=\alpha^{*}-u_{0}^{ \pm}(s, 0), \quad v_{0}^{ \pm}=v^{*} \quad s \in \Gamma^{0},
$$

$k \geq 1$

$$
\phi_{k}^{ \pm}(s, 0)=-u_{k}^{ \pm}(s, 0), \quad v_{k}^{ \pm}(s, 0)=\beta_{k}(s)-\psi_{k-2}^{ \pm}(s, 0) \quad s \in \Gamma^{0} .
$$

In this way, we obtain the formal asymptotic solution of $(2.13)_{ \pm}$. Since $\left(U^{\varepsilon}, V^{\varepsilon}\right)$ is a stationary solution of $(2.6),\left(U_{ \pm}^{\varepsilon}, V_{ \pm}^{\varepsilon}\right)$ must satisfy the $C^{1}$-matching conditions; that is,

$$
\varepsilon \frac{\partial U_{+}^{\varepsilon}}{\partial \nu}=\varepsilon \frac{\partial U_{-}^{\varepsilon}}{\partial \nu}, \quad \varepsilon \frac{\partial V_{+}^{\varepsilon}}{\partial \nu}=\varepsilon \frac{\partial V_{-}^{\varepsilon}}{\partial \nu} \text { on } \Gamma^{0}
$$

After some computation, we have

$$
k=0
$$

$$
\left(v_{0}^{+}\right)_{\tau}(s, 0)=\left(v_{0}^{-}\right)_{\tau}(s, 0), \quad \dot{\phi}_{0}^{+}(s, 0)=\dot{\phi}_{0}^{-}(s, 0), \quad s \in \Gamma^{0} .
$$

$k \geq 1$

$$
\begin{aligned}
& \left(v_{k}^{+}\right)_{\tau}(s, 0)+\dot{\psi}_{k-1}^{+}(s, 0)=\left(v_{k}^{-}\right)_{\tau}(s, 0)+\dot{\psi}_{k-1}^{-}(s, 0), \\
& \dot{\phi}_{k}^{+}(s, 0)+\left(u_{k-1}^{+}\right)_{\tau}(s, 0)=\dot{\phi}_{k}^{-}(s, 0)+\left(u_{k-1}^{-}\right)_{\tau}(s, 0) .
\end{aligned}
$$

The second equation of (2.15) with the boundary and $C^{1}$-matching conditions (see (2.18) and (2.19)) is called the reduced problem; namely,

$$
\begin{cases}D \Delta v_{0}^{ \pm}+g\left(h_{ \pm}\left(v_{0}^{ \pm}\right), v_{0}^{ \pm}\right)=0 & \text { in } \Omega_{0}^{ \pm} \\ v_{0}^{ \pm}=v^{*}, \quad \frac{\partial v_{0}^{+}}{\partial \nu}=\frac{\partial v_{0}^{-}}{\partial \nu} & \text { on } \Gamma^{0} \\ \frac{\partial v_{0}^{-}}{\partial n}=0 & \text { on } \partial \Omega .\end{cases}
$$

This is a free boundary problem for $\Gamma^{0}$ which determines the asymptotic configuration of stationary interfacial patterns. We define $v_{0}(x) \in C^{1}(\bar{\Omega})$ by

$$
v_{0}(x)= \begin{cases}v_{0}^{+}(x), & x \in \Omega_{0}^{+}, \\ v_{0}^{-}(x), & x \in \Omega_{0}^{-} .\end{cases}
$$


We close this section by presenting a lemma on the representations of $\tilde{M}_{k}$, which will become useful in the next section. The proof is delegated to [6].

LEMma 2.2. $\tilde{M}_{0}, \tilde{M}_{1}$, and $\tilde{M}_{2}$ have the following forms:

$$
\begin{aligned}
\tilde{M}_{0} \equiv & \frac{\partial^{2}}{\partial \xi^{2}}, \quad \tilde{M}_{1} \equiv(N-1) H_{0}(s) \frac{\partial}{\partial \xi}, \\
\tilde{M}_{2} \equiv & \Delta^{\Gamma^{0}}-\left(P_{1}(s)+P_{2}(s)\right) \frac{\partial}{\partial \xi}+P_{3}(s) \frac{\partial^{2}}{\partial \xi^{2}} \\
& -D_{s} \frac{\partial}{\partial \xi}-H_{1}(s)\left(\xi+\gamma_{1}(s)\right) \frac{\partial}{\partial \xi},
\end{aligned}
$$

where

$$
\begin{aligned}
& P_{1}(s)=\frac{1}{2 G} \sum_{i=1}^{N-1} G_{s^{i}} \sum_{j=1}^{N-1} G^{i j} \partial_{s^{j}} \gamma_{1}, P_{2}(s)=\sum_{i=1}^{N-1} \sum_{j=1}^{N-1}\left[G_{s^{i}}^{i j} \partial_{s^{j}} \gamma_{1}+G^{i j} \partial_{s^{i} s^{j}} \gamma_{1}\right], \\
& P_{3}(s)=\sum_{i=1}^{N-1} \sum_{j=1}^{N-1} G^{i j} \partial_{s^{i}} \gamma_{1} \partial_{s^{j}} \gamma_{1}>0, D_{s}=\sum_{i=1}^{N-1} \sum_{j=1}^{N-1} G^{i j}\left(\partial_{s^{i}} \gamma_{1} \frac{\partial}{\partial s^{j}}+\partial_{s^{j}} \gamma_{1} \frac{\partial}{\partial s^{i}}\right), \\
& H_{1}(s) \equiv \sum_{i=1}^{N-1} \kappa_{i}(s)^{2} .
\end{aligned}
$$

$H_{0}(s)\left(\right.$ resp., $\left.\kappa_{i}(s)\right)$ are the mean (resp., principal) curvature of $\Gamma^{0}$ at $s \in \Gamma^{0}, G^{i j}$ is the contravariant metric tensor for the manifold $\Gamma^{0}$ of dimension $N-1, G=\operatorname{det}\left(G^{i j}\right)$, and $\Delta^{\Gamma^{0}}$ is Laplace-Beltrami's operator defined on $\Gamma^{0}$. In particular, the coefficients of $\frac{\partial}{\partial s^{j}}$ in $D_{s}$ are independent of $\xi$.

3. Instability result for stationary patterns as $\varepsilon \downarrow 0$. In this section we prove that the internal layered solutions in the previous section must become unstable when $\varepsilon$ tends to zero. For this purpose, we show that the linearized eigenvalue problem around $\left(U^{\varepsilon}, V^{\varepsilon}\right)$,

$$
\left\{\begin{array}{l}
\lambda w=\varepsilon^{2} M^{\varepsilon} w+f_{u}^{\varepsilon} w+f_{v}^{\varepsilon} z, \\
\lambda z=D M^{\varepsilon} z+g_{u}^{\varepsilon} w+g_{v}^{\varepsilon} z
\end{array}\right.
$$

has an unstable eigenvalue where $\lambda$ is the eigenvalue parameter, where $f_{u}^{\varepsilon}=\frac{\partial}{\partial u} f\left(U^{\varepsilon}, V^{\varepsilon}\right)$ and so on. The main result is the following.

TheOREM 3.1. Suppose that (1.1) has an $\varepsilon$-family of stationary matched asymptotic solutions of order 1 (i.e., $m=1$ ) whose interface is smooth up to $\varepsilon=0$. Then, (3.1) has a positive (i.e., unstable) eigenvalue of $O(\varepsilon)$ for small $\varepsilon$.

Proof. We divide the proof into three steps. In the first two steps we assume that the $\varepsilon$-family of stationary solutions has an asymptotic expansion up to order 2 (i.e., $m=2$ ), which makes the proof readable, especially to understand how to handle the Laplacian part. Then it is easy to modify the proof to be valid for the order 1 case (see Step 3 below). Apparently (3.1) is not a self-adjoint problem, since $f_{v}^{\varepsilon} \neq g_{u}^{\varepsilon}$. In order to reduce the problem to a self-adjoint one, we first introduce the following 
auxiliary problem:

$$
\left\{\begin{array}{l}
\lambda w=\varepsilon^{2} M^{\varepsilon} w+f_{u}^{\varepsilon} w+f_{v}^{\varepsilon} z, \\
\eta z=D M^{\varepsilon} z+g_{u}^{\varepsilon} w+g_{v}^{\varepsilon} z,
\end{array}\right.
$$

where $\eta$ is an auxiliary parameter. The following Step 1 deals with the case $\eta=0$, where, by solving the second equation with respect to $z$ (see (A.6)) and substituting it into the first equation, we have a self-adjoint problem of $w$. Then the existence of the unstable eigenvalue for (3.2) with $\eta=0$ is shown via the variational method. This result can be extended to case $\eta>0$ in Step 2, where it is proved that a positive eigenvalue of $O(\varepsilon)$ of (3.2) exists for each $\eta \neq 0$. The proof of Theorem 3.1 for $m=2$ is an immediate consequence of these two steps. Finally in Step 3 we show that the expansion up to order 1 is sufficient for the proof of instability. Note that the linearized instability implies a nonlinear one for the class of evolutional systems like (1.1).

Step 1 ( $\eta=0$ case). Solving the second equation of (3.2) with $\eta=0$ with respect to $z$ as

$$
z=\left(N^{\varepsilon}\right)^{-1} g_{u}^{\varepsilon} w,
$$

where $\left(N^{\varepsilon}\right)^{-1} \equiv\left(-D M^{\varepsilon}-g_{v}^{\varepsilon}\right)^{-1}$, which is well defined from (A.6), and then substituting (3.3) into the first equation of (3.2), we obtain a scalar problem for $w$ :

$$
\lambda w=\varepsilon^{2} M^{\varepsilon} w+f_{u}^{\varepsilon} w+f_{v}^{\varepsilon}\left(N^{\varepsilon}\right)^{-1} g_{u}^{\varepsilon} w .
$$

From (A.1), (3.4) becomes a self-adjoint problem.

Lemma 3.2. (3.4) has a positive (i.e., unstable) eigenvalue of $O(\varepsilon)$ for small $\varepsilon$.

Proof. In what follows, for simplicity of notation, we can assume that $f_{v} \equiv-1$ and $g_{u} \equiv 1$ without loss of generality. Since the linearized operator (3.4) is self-adjoint, we shall prove that the largest eigenvalue $\lambda_{0}^{\varepsilon}$ of (3.4) becomes positive for small $\varepsilon$, which is characterized by

$$
\lambda_{0}^{\varepsilon}=\sup _{w \in H^{1}(\Omega)} \frac{\int_{\Omega}\left\{-\varepsilon^{2}\left|\nabla_{M^{\varepsilon}} w\right|^{2}+f_{u}^{\varepsilon} w^{2}-\left|\left(N^{\varepsilon}\right)^{-1 / 2} w\right|^{2}\right\} d x}{\int_{\Omega} w^{2} d x},
$$

where $\nabla_{M^{\varepsilon}}$ is the representation of $\nabla$ with respect to the coordinate $\hat{x}$ (see section 2 ). Recall that we write $x$ instead of $\hat{x}$ and hence $Y(x)=\tau$ and $\xi \equiv \tau / \varepsilon$. Now we construct a suitable test function for our purpose. Let

$$
Q(\xi)= \begin{cases}\omega\left(\frac{\varepsilon \xi}{d}\right) \dot{U}(\xi) & \text { for }|\xi| \leq \frac{d}{\varepsilon} \\ 0 & \text { for }|\xi| \geq \frac{d}{\varepsilon}\end{cases}
$$

where $U$ is a solution of

$$
\left\{\begin{array}{l}
\ddot{U}+f\left(U, v^{*}\right)=0, \\
U( \pm \infty)=h_{\mp}\left(v^{*}\right), \quad U(0)=\alpha^{*} .
\end{array}\right.
$$


We define $w(x)$ by the following product with $\Theta \in L^{2}\left(\Gamma^{0}\right)$ and $\|\Theta\|_{L^{2}\left(\Gamma^{0}\right)}=1$ :

$$
w(x)=\left\{\begin{array}{lr}
Q\left(\frac{Y(x)}{\varepsilon}\right) \Theta(S(x)), & x \in U_{d}\left(\Gamma^{0}\right), \\
0, & x \in \Omega \backslash U_{d}\left(\Gamma^{0}\right) .
\end{array}\right.
$$

For this $w(x), \varepsilon^{2}\left|\nabla_{M^{\varepsilon}} w\right|^{2}$ is computed as

$$
\begin{gathered}
\varepsilon^{2}\left|\nabla_{M} \varepsilon\right|^{2}=\varepsilon^{2}\left(\dot{U}^{2}\left|\nabla^{\Gamma^{0}} \Theta\right|^{2}-2 \ddot{U}^{2} \dot{U}^{2} \nabla^{\Gamma^{0} \Theta} \Theta \cdot \nabla^{\Gamma^{0}} \gamma_{1}+\ddot{U}^{2}\left|\nabla^{\Gamma^{0}} \gamma_{1}\right|^{2}\right) \\
+\ddot{U}^{2} \Theta^{2}+O\left(\varepsilon^{3}\right)
\end{gathered}
$$

in $U_{d}\left(\Gamma^{0}\right)$, where

$$
\nabla^{\Gamma^{0}} \Theta_{1} \cdot \nabla^{\Gamma^{0}} \Theta_{2} \equiv \sum_{i, j=1}^{N-1} G^{i j} \frac{\partial \Theta_{1}}{\partial s^{i}} \frac{\partial \Theta_{2}}{\partial s^{j}}, \quad\left|\nabla^{\Gamma^{0}} \Theta_{3}\right|^{2} \equiv \nabla^{\Gamma^{0}} \Theta_{3} \cdot \nabla^{\Gamma^{0}} \Theta_{3}
$$

for $\Theta_{i} \in L^{2}\left(\Gamma^{0}\right)(i=1,2,3)$, and $G^{i j}$ is the contravariant metric tensor for $\Gamma^{0}$. Here we used the fact that

$$
\left|\nabla_{M^{\varepsilon}} \hat{u}\right|^{2}=\left|\nabla_{(s, y)} u\right|^{2} \equiv \sum_{i, j=1}^{N-1} g^{i j} \frac{\partial u}{\partial s^{i}} \frac{\partial u}{\partial s^{j}}+\left(\frac{\partial u}{\partial y}\right)^{2}
$$

for $\hat{u}(s, \tau)=u(s, y)$. Hence, for a function $\varphi=\varphi(s, \tau(s, y, \varepsilon) / \varepsilon)$, we note that $\frac{\partial \varphi}{\partial s^{i}}=$ $\varphi_{s^{i}}+\frac{1}{\varepsilon} \varphi_{\xi} \tau_{s^{i}}, \frac{\partial \varphi}{\partial y}=\frac{1}{\varepsilon} \varphi_{\xi} \tau_{y}, \tau_{s^{i}}=-\varepsilon \partial_{s^{i}} \gamma_{1}+O\left(\varepsilon^{2}\right)$, and $\tau_{y} \equiv 1$ in a neighborhood of $\tau=0$ (see (2.5)). Integrating (3.7) over $\Omega$, the first term of numerator of (3.5) becomes

$$
\begin{gathered}
\int_{\Omega} \varepsilon^{2}\left|\nabla_{M^{\varepsilon}} w\right|^{2} d x \\
=\varepsilon \int_{\Gamma^{0}} \int_{|\xi| \leq \frac{d}{\varepsilon}}\left\{\varepsilon^{2} \dot{U}^{2}\left|\nabla^{\Gamma^{0}} \Theta\right|^{2}+\varepsilon^{2} \ddot{U}^{2} \hat{P}_{1}(s) \Theta^{2}+\ddot{U}^{2} \Theta^{2}\right\} d \xi d S+O\left(\varepsilon^{4}\right),
\end{gathered}
$$

where $\hat{P}_{1}(s)=\left|\nabla^{\Gamma^{0}} \gamma_{1}\right|^{2}$. The remainder term $O\left(\varepsilon^{4}\right)$ depends only on $\Gamma^{0}$ and the $L^{2}$-norm of $\Theta$. In order to compute the second term of the numerator of (3.5), first note that

$$
f_{u}^{\varepsilon}=\left\{\begin{array}{l}
\tilde{F}_{u}^{0+}+\varepsilon \tilde{F}_{u}^{1+}+\varepsilon^{2} \tilde{F}_{u}^{2+}+O\left(\varepsilon^{3}\right) \text { in } \Omega_{0}^{+} \cap U_{d}\left(\Gamma^{0}\right), \\
\tilde{F}_{u}^{0-}+\varepsilon \tilde{F}_{u}^{1-}+\varepsilon^{2} \tilde{F}_{u}^{2-}+O\left(\varepsilon^{3}\right) \text { in } \Omega_{0}^{-} \cap U_{d}\left(\Gamma^{0}\right),
\end{array}\right.
$$

where

$$
\begin{aligned}
& \tilde{F}_{u}^{0 \pm} \equiv f_{u}\left(h_{ \pm}\left(v^{*}\right)+\phi_{0}^{ \pm}, v^{*}\right), \\
& \tilde{F}_{u}^{1 \pm} \equiv f_{u u}\left(h_{ \pm}\left(v^{*}\right)+\phi_{0}^{ \pm}, v^{*}\right)\left\{\xi\left(u_{0}^{ \pm}\right)_{\tau}(s, 0)+u_{1}^{ \pm}(s, 0)+\phi_{1}^{ \pm}\right\} \\
& \quad+f_{u v}\left(h_{ \pm}\left(v^{*}\right)+\phi_{0}^{ \pm}, v^{*}\right)\left\{\xi\left(v_{0}\right)_{\tau}(s, 0)+v_{1}^{ \pm}(s, 0)\right\},
\end{aligned}
$$


and the remainder term $O\left(\varepsilon^{3}\right)$ depends only on the stationary pattern $\left(U^{\varepsilon}, V^{\varepsilon}\right)$. The $O(1)$ term of (3.9) multiplied by $w^{2}$ combined with the third term of (3.8) vanishes, which is easily seen by differentiating (3.6) with respect to $\xi$. Hence we only focus on the contribution of (3.9) coming from the $O(\varepsilon)$-term and higher. The next equality is a key ingredient for the proof

$$
\int_{-\infty}^{0} \tilde{F}_{u}^{1+} \dot{U}^{2} d \xi+\int_{0}^{\infty} \tilde{F}_{u}^{1-} \dot{U}^{2} d \xi=\left(v_{0}\right)_{\tau}(s, 0) \frac{d}{d v} J\left(v^{*}\right)>0 .
$$

In order to show (3.10), we note that $\dot{U}=\dot{\phi}_{0}^{ \pm}, \ddot{U}=\ddot{\phi}_{0}^{ \pm}$for $\xi \in I^{\mp}$ (so we omit the superscript \pm of $\dot{\phi}_{0}$ and $\ddot{\phi}_{0}$ ), and $p^{ \pm} \equiv \dot{\phi}_{1}^{ \pm}$satisfy the next equation (see section 2):

$$
\ddot{p}^{ \pm}+\tilde{F}_{u}^{0 \pm} p^{ \pm}=\Omega^{ \pm}
$$

where

$$
\Omega^{ \pm}(s, \xi) \equiv H^{ \pm}(s, \xi)-\left\{\left(u_{0}^{ \pm}\right)_{\tau}(s, 0) \tilde{f}_{u}^{0 \pm}+\left(v_{0}\right)_{\tau}(s, 0) \tilde{f}_{v}^{0 \pm}\right\}
$$

and

$$
H^{ \pm} \equiv-(N-1) H_{0} \ddot{\phi}_{0}-\tilde{F}_{u}^{1 \pm} \dot{\phi}_{0} .
$$

Multiplying $\dot{\phi}_{0}$ on both sides of (3.11) and using the relations

$$
\int_{\mp \infty}^{0} \tilde{f}_{u}^{0 \pm} \dot{\phi}_{0}(z) d z=-\ddot{\phi}_{0}(0), \quad \int_{\mp \infty}^{0} \tilde{f}_{v}^{0 \pm} \dot{\phi}_{0}(z) d z=\int_{h_{ \pm}\left(v^{*}\right)}^{\alpha^{*}} f_{v}\left(u, v^{*}\right) d u,
$$

we obtain, by integration by parts,

$$
\begin{gathered}
\int_{\mp \infty}^{0} H^{ \pm}(s, z) \dot{\phi}_{0}(z) d z=\ddot{\phi}_{1}^{ \pm}(s, 0) \dot{\phi}_{0}(0)-\ddot{\phi}_{0}(0)\left\{\dot{\phi}_{1}^{ \pm}(s, 0)+\left(u_{0}^{ \pm}\right)_{\tau}(s, 0)\right\} \\
+\left(v_{0}\right)_{\tau}(s, 0) \int_{h_{ \pm}\left(v^{*}\right)}^{\alpha^{*}} f_{v}\left(u, v^{*}\right) d u .
\end{gathered}
$$

On the other hand, multiplying $\dot{\phi}_{0}$ on both sides of (3.12) and using (3.13), we have

$$
\begin{aligned}
& \int_{-\infty}^{0} \tilde{F}_{u}^{1+} \dot{U}^{2} d \xi+\int_{0}^{\infty} \tilde{F}_{u}^{1-} \dot{U}^{2} d \xi=\int_{-\infty}^{0} \tilde{F}_{u}^{1+} \dot{\phi}_{0}^{2} d \xi+\int_{0}^{\infty} \tilde{F}_{u}^{1-} \dot{\phi}_{0}^{2} d \xi \\
& =-(N-1) H_{0} \int_{-\infty}^{0} \ddot{\phi}_{0} \dot{\phi}_{0} d \xi-(N-1) H_{0} \int_{0}^{\infty} \ddot{\phi}_{0} \dot{\phi}_{0} d \xi \\
& -\ddot{\phi}_{1}^{+}(s, 0) \dot{\phi}_{0}(0)+\ddot{\phi}_{0}\left\{\dot{\phi}_{1}^{+}(s, 0)+\left(u_{0}^{+}\right)_{\tau}(s, 0)\right\}-\left(v_{0}\right)_{\tau}(s, 0) \int_{h_{+}\left(v^{*}\right)}^{\alpha^{*}} f_{v}\left(u, v^{*}\right) d u \\
& +\ddot{\phi}_{1}^{-}(s, 0) \dot{\phi}_{0}(0)-\ddot{\phi}_{0}\left\{\dot{\phi}_{1}^{-}(s, 0)+\left(u_{0}^{-}\right)_{\tau}(s, 0)\right\}+\left(v_{0}\right)_{\tau}(s, 0) \int_{h_{-}\left(v^{*}\right)}^{\alpha^{*}} f_{v}\left(u, v^{*}\right) d u \\
& =\left(v_{0}\right)_{\tau}(s, 0) \int_{h_{-}\left(v^{*}\right)}^{h_{+}\left(v^{*}\right)} f_{v}\left(u, v^{*}\right) d u
\end{aligned}
$$


which is the required result (3.10). Here we used the fact that $\ddot{\phi}_{1}^{+}(s, 0)=\ddot{\phi}_{1}^{-}(s, 0)$ and the $C^{1}$-matching condition of $\phi_{1}^{ \pm}$(see (2.20)). Using (3.8), (3.9), (3.10), and the Hopf boundary Lemma for $v^{0}$ on $\Gamma^{0}$ (see (1.2) and (2.21)), we obtain

$$
\begin{aligned}
\lambda_{0}^{\varepsilon} \geq C & {\left[\varepsilon \frac{m_{*}}{K_{1}} \frac{d}{d v} J\left(v^{*}\right)\right.} \\
& +\int_{\Gamma} \varepsilon^{2}\left\{-\left|\nabla^{\Gamma^{0} \Theta}\right|^{2}-\frac{1}{K_{1}}\left(K_{2} \hat{P}_{1}(s)-\hat{P}_{2}(s)\right) \Theta^{2}\right\} d S \\
& \left.-\frac{1}{K_{1} \varepsilon} \int_{\Omega}\left|\left(N^{\varepsilon}\right)^{-1 / 2} w\right|^{2} d x\right]+O\left(\varepsilon^{3}\right),
\end{aligned}
$$

where

$$
\begin{gathered}
m_{*} \equiv \min _{s \in \Gamma^{0}}\left(v_{0}\right)_{\tau}(s, 0)<0, \quad K_{1} \equiv \int_{-\infty}^{\infty} \dot{U}^{2} d \xi, \quad K_{2} \equiv \int_{-\infty}^{\infty} \ddot{U}^{2} d \xi \\
\hat{P}_{2}(s) \equiv \int_{-\infty}^{0} \tilde{F}_{u}^{2+} \dot{U}^{2} d \xi+\int_{0}^{\infty} \tilde{F}_{u}^{2-} \dot{U}^{2} d \xi
\end{gathered}
$$

and $C$ is a positive constant. The objective is to choose an appropriate test function in order to make the first term of [ $\cdot]$ in (3.14) dominant, which is positive and $O(\varepsilon)$. First we choose $\Theta$ as the $k$ th eigenfunction $\Theta_{k}$ of the following eigenvalue problem:

$$
\Delta^{\Gamma} \Theta_{k}-\frac{1}{K_{1}}\left(K_{2} \hat{P}_{1}(s)-\hat{P}_{2}(s)\right) \Theta_{k}=\mu_{k} \Theta_{k} \quad \text { on } \Gamma^{0} .
$$

Then the second term of (3.14) is equal to $\varepsilon^{2} \mu_{k}$. Note also that $\Theta_{k}$ converges to 0 as $k \rightarrow \infty$ in weak $L^{2}\left(\Gamma^{0}\right)$-sense. As for the third term of (3.14), which comes from the nonlocal part, we first note that when $\varepsilon$ tends to zero,

$$
\frac{1}{\varepsilon} Q\left(\frac{\tau}{\varepsilon}\right)=\frac{1}{\varepsilon} \dot{U}\left(\frac{\tau}{\varepsilon}\right) \omega\left(\frac{\tau}{d}\right) \longrightarrow c_{0} \delta(\tau) \quad \text { in } H^{-1}((-d, d)) \text {-sense, }
$$

where $\delta(\tau)$ is a Dirac's $\delta$-function at 0 and $c_{0}$ is a positive constant. Let $K_{k}^{\varepsilon}$ be

$$
K_{k}^{\varepsilon} \equiv \int_{\Omega}\left|\left(N^{\varepsilon}\right)^{-1 / 2}\left(\frac{w_{k}}{\varepsilon}\right)\right|^{2} d x=\int_{\Omega}\left[\left(N^{\varepsilon}\right)^{-1}\left(\frac{w_{k}}{\varepsilon}\right)\right]\left(\frac{w_{k}}{\varepsilon}\right) d x .
$$

In view of (3.15) and considering that $\left(N^{\varepsilon}\right)^{-1}$ is a uniformly bounded operator mapping from $H^{-1}(\Omega)$ to $H^{1}(\Omega)$ with respect to $\varepsilon$, we see that $K_{k}^{\varepsilon}$ is uniformly bounded with respect to $\varepsilon$ and $k$ and that $\int_{-d}^{d}(Q(\tau / \varepsilon) / \varepsilon) \cdot d \tau$ converges to the trace operator on $\Gamma^{0}$ from $H^{1}(\Omega)$ to $H^{1 / 2}\left(\Gamma^{0}\right)$ in operator norm sense. Therefore, by using the fact that $\Theta_{k}$ converges weakly to 0 as $k \rightarrow \infty$, we see that for any given small $c^{*}>0$, there exists an $\varepsilon_{0}$ and $k_{0}$ such that

$$
K_{k}^{\varepsilon}<c^{*} \text { for } 0<\varepsilon \leq \varepsilon_{0}, k \geq k_{0} .
$$

Substituting $\Theta=\Theta_{k}$ and $w=w_{k}$ into (3.14), we have

$$
\lambda_{0}^{\varepsilon} \geq C \varepsilon\left[\frac{m_{*}}{K_{1}} \frac{d}{d v} J\left(v^{*}\right)-\frac{1}{K_{1}} K_{k}^{\varepsilon}+\varepsilon \mu_{k}\right]+O\left(\varepsilon^{3}\right),
$$


where $C$ is a positive constant. Using (3.16) and taking $\varepsilon$ smaller, if necessary, we see that

$$
\frac{m_{*}}{K_{1}} \frac{d}{d v} J\left(v^{*}\right)-\frac{1}{K_{1}} K_{k}^{\varepsilon}>0 .
$$

Therefore the right-hand side of (3.5) becomes positive for sufficiently small $\varepsilon>0$, which is greater or equal to the $O(\varepsilon)$ quantity. In the rest of the proof, we show that the upper bound of $(3.5)$ is also of $O(\varepsilon)$. Since the first and the third terms of the numerator of (3.5) are nonpositive, it holds obviously that

$$
\lambda_{0}^{\varepsilon} \leq \sup _{w \in H^{1}(\Omega)} \frac{\int_{\Omega} f_{u}^{\varepsilon} w^{2} d x}{\int_{\Omega} w^{2} d x} .
$$

In view of the expansion (3.9) and the assumptions (A.2) and (A.4), $f_{u}^{\varepsilon}$ has a positive sign only in the $\varepsilon$-neighborhood along the normal direction of $\Gamma^{0}$. Therefore we have the estimate

$$
\lambda_{0}^{\varepsilon} \leq C \varepsilon\left|\Gamma^{0}\right|
$$

where $C$ is a positive constant and $|\cdot|$ denotes the area, which completes the proof of Lemma 3.2.

Step $2(\eta \neq 0$ case). Rewriting $(3.2)$ as

$$
\left\{\begin{array}{l}
\lambda w=\varepsilon^{2} M^{\varepsilon} w+f_{u}^{\varepsilon} w+f_{v}^{\varepsilon} z \\
0=D M^{\varepsilon} z+g_{u}^{\varepsilon} w+\left(g_{v}^{\varepsilon}-\eta\right) z
\end{array}\right.
$$

and noting that $g_{v}^{\varepsilon}-\eta<0$ for $\eta \geq 0$ from (A.6), we see that all the computation in Step 1 is also valid for (3.18) with $\eta \geq 0$. Therefore we have the following lemma.

LEMmA 3.3. (3.18) has a positive eigenvalue $\lambda=\lambda^{\varepsilon}(\eta)$ for $\eta \geq 0$. Moreover, there exist positive constants $C_{0}$ and $C_{1}\left(C_{0}<C_{1}\right)$ which are independent of $\eta$ and $\varepsilon$ such that

$$
C_{0} \varepsilon<\lambda^{\varepsilon}(\eta)<C_{1} \varepsilon
$$

holds for $\eta \geq 0$.

Proof of Theorem 3.1 ( $m=2$ case). It follows from Lemma 3.3 that $\lambda^{\varepsilon}(\eta)$ is a continuous function of $\eta$ for $\eta \geq 0$. Since $\lambda^{\varepsilon}(\eta)$ has lower and upper bounds like (3.19), we see that $\eta=\lambda^{\varepsilon}(\eta)$ holds at least at one point $\eta=\eta^{*}(>0)$ by the intermediate value theorem. This $\eta^{*}$ is the required unstable eigenvalue for (3.1).

Step 3 (extension to $m=1$ case). It suffices to show how Step 1 should be changed under the weaker assumption. When the asymptotic expansion has only terms up to $m=1$, we see from Lemma 2.2 that we lose the precise expression for the gradient part $\left|\nabla_{M \varepsilon} w\right|^{2}$, and the right-hand side of (3.7) becomes $\ddot{U}^{2} \Theta^{2}+O\left(\varepsilon^{2}\right)$; namely, the gradient part is part of the remainder term $O\left(\varepsilon^{2}\right)$. Similarly, the first two terms of the integrand of the right-hand side of (3.8) move to the remainder term $O\left(\varepsilon^{3}\right)$. On the other hand, (3.10) remains valid. Hence (3.14) becomes the following:

$$
\lambda_{0}^{\varepsilon} \geq C\left[\varepsilon \frac{m_{*}}{K_{1}} \frac{d}{d v} J\left(v^{*}\right)-\frac{1}{K_{1} \varepsilon} \int_{\Omega}\left|\left(N^{\varepsilon}\right)^{-1 / 2} w\right|^{2} d x\right]+O\left(\varepsilon^{2}\right) .
$$


Note that the remainder term $O\left(\varepsilon^{2}\right)$ depends on $\Theta$. In order to get the estimate (3.16), we select the function $\Theta$ in Step 1 as an eigenfunction of the elliptic operator of second order. However it is not necessary to take such an eigenfunction; in fact it suffices to employ any weakly convergent sequence to zero. Once we fix an appropriate $\Theta$ and then take $\varepsilon$ to be sufficiently small, we have a similar estimate to (3.17). It is easy to see that Lemma 3.3 and the proof after it hold true for this case. This completes the proof of Theorem 3.1.

4. Concluding remarks. As was mentioned in section 1, Theorem 1.1 strongly suggests that stable patterns of (1.1) become very fine and/or complicated in the limit of $\varepsilon \downarrow 0$ in higher dimensional spaces. What we discuss here seeks to find an appropriate scaling in space and time by which the resulting singular limit dynamics could have stable patterns of finite size. Such patterns are usually maintained by the balance of two competing forces as described below. In the course of the following formal analysis, it is intuitive why the stable patterns of (1.1) must become fine in the original scale.

Suppose there is a sharp transition layer (interface) $\Gamma$ connecting two stable bulk states. There are two forces that drive the interface: one is the bulk force causing the translation of interface with certain speed $W\left(\left.v\right|_{\Gamma}\right)$ which depends on the value of $v$ at $\Gamma$; the other is a geometric force, i.e., mean-curvature effect.

In one word, the characteristic size of stable patterns is determined by the balance between the above two forces and turns out to be proportional to $\varepsilon^{1 / 3}$. It should be noted that the scale $\varepsilon^{1 / 3}$ coincides with the fastest growing wavelength of the planar front of (1.1) (see [7]). In what follows we consider a smooth subdomain $\tilde{\Omega}_{\varepsilon}(\subset \Omega)$ and assume that both $u$ and $v$ satisfy the Neumann boundary conditions on $\partial \tilde{\Omega}_{\varepsilon}$ and the diameter of $\tilde{\Omega}_{\varepsilon}$ shrinks to zero as $\varepsilon \downarrow 0$ with order $\varepsilon^{\alpha}$. Here $\alpha(0<\alpha<1)$ is an unknown exponent. Typically $\tilde{\Omega}_{\varepsilon}$ is a unit cell of some periodic structure in $\mathbf{R}^{N}$.

Applying a change of variable with unknown exponent $\alpha$

$$
\mathbf{y}=\frac{\mathbf{x}}{\varepsilon^{\alpha}} \quad(0<\alpha<1)
$$

to (1.1) ( $D=1$ for simplicity), we have

$$
\left\{\begin{array}{l}
u_{t}=\varepsilon^{2(1-\alpha)} \Delta_{\mathbf{y}} u+f(u, v) \\
v_{t}=\varepsilon^{-2 \alpha} \Delta_{\mathbf{y}} v+g(u, v)
\end{array} \quad \text { in } \hat{\Omega}_{\varepsilon},\right.
$$

where $\Delta_{\mathbf{y}}$ stands for the Laplacian in $\mathbf{y}$-variable and $\hat{\Omega}_{\varepsilon}$ is the stretched domain of $\tilde{\Omega}_{\varepsilon}$. It is more convenient to rewrite (4.1) in the following form:

$$
\left\{\begin{array}{l}
\varepsilon^{-(1-\alpha)} u_{t}=\varepsilon^{1-\alpha} \Delta_{\mathbf{y}} u+\varepsilon^{-(1-\alpha)} f(u, v), \\
\varepsilon^{2 \alpha} v_{t}=\Delta_{\mathbf{y}} v+\varepsilon^{2 \alpha} g(u, v)
\end{array}\right.
$$

Suppose $\hat{\Omega}_{\varepsilon}$ has a smooth limit $\hat{\Omega}$ as $\varepsilon \downarrow 0$. Taking a limiting procedure similar to [3], we obtain the following interfacial dynamics:

$$
\begin{cases}\varepsilon^{-(1-\alpha)} \Gamma_{t}=\left\{W\left(\left.v\right|_{\Gamma}\right)-\varepsilon^{1-\alpha} \kappa\right\} \mathbf{N} & \text { on } \Gamma(t), \\ \varepsilon^{2 \alpha} v_{t}^{ \pm}=\Delta_{\mathbf{y}} v^{ \pm}+\varepsilon^{2 \alpha} g\left(h^{ \pm}\left(v^{ \pm}\right), v^{ \pm}\right) & \text {in } \hat{\Omega}^{ \pm}(t),\end{cases}
$$


where $\Gamma(t)$ stands for the limiting configuration of the interface, $\kappa$ denotes the mean curvature of $\Gamma(t), \mathbf{N}$ is the unit normal vector at $\Gamma$ pointing from $\hat{\Omega}^{+}$to $\hat{\Omega}^{-}, W(\cdot)$ is the travelling velocity of the first equation of (1.1) with $\varepsilon=1$ for a fixed $v$ and is typically a monotone decreasing function of $v$, the domain $\hat{\Omega}$ is divided into two parts $\bar{\Omega}^{ \pm}(t)$ by $\Gamma(t)$ where $u=h^{ \pm}(v)$ on each subdomain, respectively, and $v$ is matched in $C^{1}$-sense at $\Gamma(t)$. In view of the second equation of (4.3), $v^{ \pm}$may be expanded as

$$
v^{ \pm}=v_{0}^{ \pm}(\mathbf{y}, t)+\varepsilon^{2 \alpha} v_{1}^{ \pm}(\mathbf{y}, t)+O\left(\varepsilon^{4 \alpha}\right) .
$$

Substituting (4.4) into (4.3) and equating like powers of $\varepsilon$, we easily see that $v_{0}^{ \pm} \equiv v^{*}$, where $v^{*}$ is the equal area level of $f(u, v)$ (see (A.3)) with $W\left(v^{*}\right)=0$. Expanding $W\left(\left.v\right|_{\Gamma}\right)$ into the Taylor series, the principal part of the next order of (4.3) becomes

$$
\left\{\begin{array}{l}
\varepsilon^{-(1-\alpha)} \Gamma_{t}=\left\{\left.\varepsilon^{2 \alpha} W^{\prime}\left(v^{*}\right) v_{1}\right|_{\Gamma}-\varepsilon^{1-\alpha} \kappa\right\} \mathbf{N} \quad \text { on } \Gamma(t), \\
0=\Delta_{\mathbf{y}} v_{1}^{ \pm}+g\left(h^{ \pm}\left(v^{*}\right), v^{*}\right) \quad \text { in } \hat{\Omega}^{ \pm}(t) .
\end{array}\right.
$$

The first term of the right-hand side of (4.5) is the bulk force and the second one is the mean-curvature effect. In order to make these two terms comparable, namely, in order that the bulk force is balanced with the curvature effect, the exponent $\alpha$ must be taken as $\alpha=\frac{1}{3}$. Suppose $\alpha \neq \frac{1}{3}$. Then either the bulk force or the curvature effect becomes dominant as $\varepsilon \downarrow 0$; hence there is no chance to have nontrivial stationary patterns of finite size in such an $\varepsilon^{\alpha}$-rescaled domain. Employing this exponent $\alpha=\frac{1}{3}$ and introducing a new time scale $\tau \equiv \varepsilon^{4 / 3} t$, the rescaled interfacial dynamics is given by

$$
\begin{cases}\Gamma_{\tau}=\left\{\left.W^{\prime}\left(v^{*}\right) v_{1}\right|_{\Gamma}-\kappa\right\} \mathbf{N} & \text { on } \Gamma(t), \\ 0=\Delta_{\mathbf{y}} v_{1}^{ \pm}+g\left(h^{ \pm}\left(v^{*}\right), v^{*}\right) & \text { in } \bar{\Omega}^{ \pm}(t) .\end{cases}
$$

Suppose $\tilde{\Omega}_{\varepsilon}$ is the unit cell of a periodic structure such as hexagonal lattice and that $\tilde{\Omega}_{\varepsilon} / \varepsilon^{1 / 3}$ has a definite limit $\hat{\Omega}$ as $\varepsilon \downarrow 0$; then the stationary problem of (4.6)

$$
\left\{\begin{array}{l}
0=\left\{\left.W^{\prime}\left(v^{*}\right) v_{1}\right|_{\Gamma}-\kappa\right\} \mathbf{N} \quad \text { on } \Gamma \\
0=\Delta_{\mathbf{y}} v_{1}^{ \pm}+g\left(h^{ \pm}\left(v^{*}\right), v^{*}\right) \quad \text { in } \hat{\Omega}^{ \pm} \\
v_{1}^{ \pm} \text {are matched in } C^{1} \text {-sense at } \Gamma
\end{array}\right.
$$

is expected to give a stable morphology of the unit cell. We call (4.7) the morphology equations of (1.1). Note that (4.7) is exactly the same as (2.19) in [6] where Suzuki used the matched asymptotic method to obtain it. However very little is known about the existence problem for (4.7) as well as their geometric profiles.

There is another observation due to [4] for a related system to (1.1) from a different point of view, that claims that the global minimizer of the following functional must oscillate rapidly with frequency being proportional to $\varepsilon^{1 / 3}$. The functional is given by

$$
\int_{\Omega}\left\{\varepsilon|\nabla u|^{2}+\frac{1}{\varepsilon} W(u)+\frac{1}{\varepsilon}\left|(-\Delta+\gamma I)^{-1 / 2} u\right|^{2}\right\} d x
$$


where $W(u)$ is a double-well potential like $u^{4} / 4-u^{2} / 2$. This is related to our problem in the following sense. Suppose the relaxation time of $v$ is much shorter than $u$ (i.e., the quasi-static assumption for $v$ is valid); then (1.1) can be replaced by

$$
\left\{\begin{array}{lr}
u_{t}=\varepsilon \Delta u+\frac{1}{\varepsilon} f_{0}(u)-\frac{1}{\varepsilon} v & (x, t) \in \Omega \times(0, \infty), \\
0=D \Delta v+u-\gamma v & (x, t) \in \partial \Omega \times(0, \infty), \\
\frac{\partial u}{\partial n}=0=\frac{\partial v}{\partial n} &
\end{array}\right.
$$

where $f_{0}(u)=u-u^{3}$. Solving the second equation with respect to $v$ and substituting it to the first equation, we have a scalar equation for $u$ with nonlocal term

$$
u_{t}=\varepsilon \Delta u+\frac{1}{\varepsilon} f_{0}(u)-\frac{1}{\varepsilon}(-\Delta+\gamma I)^{-1} u,
$$

which is the $L^{2}$-gradient equation of the functional (4.8). Suppose that $\Omega=Q=$ $(0,1)^{N}$ ( $N$-dimensional cube) with periodic boundary conditions. We see by employing the arguments of [4] that the global minimizer $u_{\varepsilon}$ of (4.8) has to satisfy the following inequality:

$$
C_{1} \varepsilon^{-1 / 3} \leq \frac{\int_{Q}\left|\nabla H\left(u_{\varepsilon}\right)\right| d x}{\int_{Q}\left|u_{\varepsilon}\right| d x} \leq C_{2} \varepsilon^{-1 / 3},
$$

where $H(z)=\int_{0}^{z} W^{1 / 2}(s) d s$ and $C_{1}, C_{2}$ are positive constants independent of $\varepsilon$. Roughly speaking, the middle term of (4.10) counts the number of interface, and hence, (4.10) means that the global minimizer has to take a fine structure, although we do not know whether $u_{\varepsilon}$ is spatially periodic or not. Finally, it should be noted that the estimate (4.10) is valid only for the global minimizer and not for the local minimizers.

\section{REFERENCES}

[1] J. Boissonade, E. Dulos, And P. De Kepper, Turing patterns: From myth to reality, in Chemical Waves and Patterns, R. Kapral and K. Showalter, eds., Kluwer Academic Publishers, Norwell, MA, 1995, pp. 221-268.

[2] H. IKEDA, On the asymptotic solutions for a weakly coupled elliptic boundary value problem with a small parameter, Hiroshima Math. J., 16 (1986), pp. 227-250.

[3] X.-Y. Chen, Dynamics of interfaces in reaction diffusion systems, Hiroshima Math. J., 21 (1991), pp. 47-83.

[4] S. Müller and G. Weiss, private communication, 1996.

[5] Y. Nishiura, Coexistence of Infinitely Many Stable Solutions to Reaction Diffusion Systems in the Singular Limit, Dynam. Report. Expositions Dynam. Systems (N.S.) 3, SpringerVerlag, New York, 1994, pp. 25-103.

[6] H. SuzukI, Asymptotic characterization of stationary interfacial patterns for reaction diffusion systems, Hokkaido Math. J., 26 (1997), pp. 631-667.

[7] M. TANiguchi and Y. Nishiura, Instability of planar interfaces in reaction-diffusion systems, SIAM J. Math. Anal., 25 (1994), pp. 99-134.

[8] M. TANiguchi And Y. Nishiura, Stability and characteristic wavelength of planar interfaces in the large diffusion limit of the inhibitor, Proc. Roy. Soc. Edinburgh Sect. A, 126 (1996), pp. 117-145.

[9] A. Turing, The chemical basis of morphogenesis, Philos. Trans. Roy. Soc. London Ser. B, 237 (1952), pp. 37-72. 